\title{
Yeni periferal tetra-(E)-4-(3-(3-(2,4,6-trimetoksifenil)akriloil)fenoksi) substitüe metalsiz ve metalli ftalosiyaninlerin sentezi ve karakterizasyonu
}

\author{
Synthesis and characterization of novel peripherally tetra-(E)-4-(3-(3-(2,4,6- \\ trimethoxyphenyl)acryloyl)phenoxy) substituted metal-free and metallophthalocyanines \\ Volkan CAKIR*1,a \\ ${ }^{\text {I} G i r e s u n ~ U ̈ n i v e r s i t e s i, ~ E s p i y e ~ M e s l e k ~ Y u ̈ k s e k ~ O k u l u ~}$ \\ Mülkiyet Koruma ve Güvenlik Bölümü, Acil Durum ve Afet Yönetimi Programı, 28200, Espiye/Giresun
}

• Geliş tarihi / Received: 09.04.2020 • Düzeltilerek geliş tarihi / Received in revised form: 26.10.2020 • Kabul tarihi / Accepted: 28.12 .2020

Öz

$\mathrm{Bu}$ çalışma kapsamında, yeni hidroksil bileşiği 1, ftalonitril bileşiği 3, periferal tetra-(E)-4-(3-(3-(2,4,6trimetoksifenil)akriloil)fenoksi) substitue metalsiz $\left(\mathrm{H}_{2} \mathrm{Pc} 4\right)$ ve metalli ftalosiyaninlerin (Co(II)Pc 5, Cu(II)Pc 6, $\mathrm{Ni}(\mathrm{II}) \mathrm{Pc}$ 7, Zn(II)Pc 8) sentezi ve karakterizasyonu gerçekleştirilmiş̧ir. 3 Bileşiğinin siklotetramerizasyon reaksiyonu sonucu yeni periferal tetra substitue metalsiz ftalosiyanin (4) elde edilmiştir. Yeni periferal tetra substitue metalli ftalosiyaninler (Co(II)Pc 5, Cu(II)Pc 6, Ni(II)Pc 7, Zn(II)Pc 8) ise 3 bileşiğinin $n$-pentanol ve 1,8-diazabisiklo[5.4.0]undek-7-en (DBU) karışımı içerisinde sırası ile susuz $\mathrm{CoCl}_{2}, \mathrm{CuCl}_{2}, \mathrm{NiCl}_{2}$ ve $\mathrm{Zn}\left(\mathrm{CH}_{3} \mathrm{COO}\right)_{2}$ tuzları ile kaynatılması sonucu sentezlenmiş̧ir. Elde edilen yeni ftalosiyanin kompleksleri kloroform, diklorometan, tetrahidrofuran, dimetilsülfoksit ve dimetilformamid gibi yaygın organik çözücüler içerisinde iyi çözünürlük göstermiş̧tir. Sentezi gerçekleştirilen yeni bileşikler $(\mathbf{1}, \mathbf{3}, \mathbf{4}, \mathbf{5}, \mathbf{6}$, 7 ve 8) IR, ${ }^{1} \mathrm{H}$ NMR, ${ }^{13} \mathrm{C}$ NMR, kütle, UV-Vis spektroskopi ve elementel analiz teknikleri kullanılarak karakterize edilmiştir.

Anahtar kelimeler: Ftalosiyanin, Karakterizasyon, Periferal, Sentez, Siklotetramerizasyon

\begin{abstract}
In this study, a new hydroxyl derivate 1, phthalonitrile derivative 3 and peripherally tetra-(E)-4-(3-(3-(2,4,6trimethoxyphenyl)acryloyl)phenoxy) substituted new metal-free 4, Co(II) 5, Cu(II) 6, Ni(II) 7 and $\mathrm{Zn}(I I) 8$ phthalocyanine derivatives were synthesized and characterized. Metal-free Pc $\mathbf{4}$ was prepared by cyclotetramerization of phthalonitrile derivate 3 and MPcs 5-8 were synthesized by heating 3 with $\mathrm{CoCl}_{2}, \mathrm{CuCl}_{2}, \mathrm{NiCl}_{2}$ and $\mathrm{Zn}\left(\mathrm{CH}_{3} \mathrm{COO}\right)_{2}$ in n-pentanol in the presence of 1,8-diazabicyclo[5.4.0]undec-7-ene (DBU), respectively. These phthalocyanines were good soluble in organic solvents such as chloroform, dichloromethane, tetrahydrofuran, dimethylsulfoxide and dimethylformamide. The new compounds $(\mathbf{1}, \mathbf{3}, \mathbf{4}, \mathbf{5}, \mathbf{6}, \mathbf{7}$ and 8 ) were characterized by a combination of $I R,{ }^{1} \mathrm{H} \mathrm{NMR},{ }^{13} \mathrm{C} N M R$, mass, UV-Vis spectroscopy and elemental analysis techniques.
\end{abstract}

Keywords: Phthalocyanine, Characterization, Peripheral, Synthesis, Cyclotetramerization

*a Volkan ÇAKIR; volkan.cakir@giresun.edu.tr, Tel: (0554) 348 83 14, orcid.org/0000-0002-5817-0817 


\section{Giriş}

Ftalosiyaninler (Pc) 18- $\pi$ elektron sistemine sahip sekiz karbon ve sekiz azot atomu içeren onaltı üyeli düzlemsel aromatik bir makro halkadan oluşur. Yapısal olarak porfirinlere benzemelerine karşın onlar gibi doğal olarak oluşmazlar ve tamamen sentetik ürünlerdir. Ftalosiyaninler siklotetramerizasyon reaksiyonu ile elde edilebilir. $\mathrm{Bu}$ reaksiyon çap olarak ftalosiyanin halka içi boşluğuna uygun metal iyonlarının varlığında daha yüksek verimle gerçekleşir $\left(\mathrm{Cu}^{2+}, \mathrm{Co}^{2+}, \mathrm{Ni}^{2+}\right.$, $\mathrm{Zn}^{+2} \ldots$ gibi) (Mutlu vd; 2020).

Metalsiz ve metalli ftalosiyaninler günümüzde oldukça fazla uygulama alanına sahiptir. Bunlardan bazılarına örnek vermek gerekirse; günümüzde bakir ftalosiyaninler boyar madde olarak kullanılabilmektedir (Yongde vd; 2006). Özellikle kobalt, demir ve rutenyum ftalosiyaninler çeşitli reaksiyonlarda katalizör olarak kullanilabilmektedir (Dini vd; 2003). Fotodinamik terapi (PDT) kanser tedavisinde kullanılmaya başlanılan ve klasik yöntemlere göre çok daha zararsız olan bir alternatiftir. Seçici olarak, tümörlü dokuyu yok eden ve fotosensitizer olarak adlandırılan bir ilaç ile ışığın kombinasyonunu kullanır. Metalli ftalosiyaninler genellikle, 650$700 \mathrm{~nm}$ civarında maksimum absorpsiyon yapmakta ve Ga, Al ve Zn ftalosiyaninler PDT'de fotosensitizer olarak kullanılmaktadır. Metalli ftalosiyaninlerden çinko ftalosiyaninler PDT için en uygun fotosensitizerlerden biridir (Kluson vd; 2008). Ftalosiyaninlerin UV spektrumları, elektron veren veya çeken gazlara maruz bırakıldıklarında değişkenlik gösterdiği için ftalosiyaninlerin kullanıldığı elektrokimyasal ve optik gaz sensörleri geliştirilmiştir (Sark1 vd; 2019). Yüksek kimyasal kararlılıklarından dolayı ftalosiyaninler, CD-ROM ve DVD-ROM teknolojilerinde de kullanım olanağı bulmuştur (Bekaroğlu; 1996).

Ftalosiyaninlerin yukarıda bahsedilen ve daha birçok uygulama alanında kullanımını kısıtlayan en büyük problem yaygın organik çözücülerdeki düşük çözünürlük veya çözünmeme problemidir (Ünlü vd; 2008). Ftalosiyaninlerin uygulama alanlarında kullanımını kısıtlayan veya düşük etkinlik çıkmasına neden olan bir diğer önemli etkense çözücü içerisinde ftalosiyaninlerin agregasyona (istiflenme) uğramasıdır. Ftalosiyanin halkasında ligandların bağlanabileceği iki farklı pozisyon bulunmaktadır, bunlar periferal ve nonperiferal olmak üzere adlandırılır. Ligandın bağlanması periferal konumdan gerçekleştiğinde çözeltide ftalosiyanin molekülleri arasındaki moleküller arası etkileşimler daha kuvvetli olur ve bu durum onların istiflenmesine (agregasyona) neden olur. Bu durumu engellemek için periferal pozisyonlara molekül ağırlığ büyük olan hacimli ligandlar bağlanarak ftalosiyanin moleküllerinin çözelti içerisinde birbirlerine fazla yaklaşması engellenerek agregasyon azaltılabilir ayrica çözünürlüğe de pozitif yönde katkı yapılmış olunur (Ao vd; 1995).

Sentez ve karakterizasyonu gerçekleştirilen hidroksil bileşiği 1, ftalonitril bileşiği $\mathbf{3}$, metalsiz ve metalli ftalosiyanin kompleksleri $4,5,6,7$ ve 8 orijinal bileşikler olup literatürde bulunmamaktadır. Çalışma kapsamında metalsiz, diamanyetik nikel(II) ve çinko(II) aynı zamanda paramanyetik kobalt(II) ve bakır(II) merkez metal iyonları içeren periferal tetra substitue ftalosiyanin kompleksleri sentezlenmiştir. Sentezi gerçekleştirilen tüm yeni bileşikler $(\mathbf{1}, \mathbf{3}, \mathbf{4}, \mathbf{5}, \mathbf{6}, \mathbf{7}$ ve 8) Infrared, ${ }^{1} \mathrm{H}$ NMR, ${ }^{13} \mathrm{C}$ NMR, MS, elektronik absorpsiyon spektroskopi ve elementel analiz teknikleri kullanılarak karakterize edilmiştir. Sentez ve karakterizasyonu gerçekleştirilen tüm kompleksler $\left(\mathbf{4}, \mathbf{5}, \mathbf{6}, 7\right.$ ve 8) kloroform $\left(\mathrm{CHCl}_{3}\right)$, diklorometan $\left(\mathrm{CH}_{2} \mathrm{Cl}_{2}\right)$, tetrahidrofuran (THF), dimetilsülfoksit (DMSO) ve dimetilformamid (DMF) gibi yaygın organik çözücüler içerisinde çözünmektedir. $\mathrm{Bu}$ çalışmada tercih edilen dimetilsülfoksit (DMSO) çözücüsü içerisinde agregasyon göstermemektedir. Bu özelliklerinden dolayı daha fazla uygulama alanı için daha farklı çalışmalarda kullanılabilecekleri düşünülmektedir.

\section{Materyal ve metot}

\subsection{Kullanilan kimyasal madde ve malzemeler}

Tüm kimyasallar yüksek kalitededir ve çeşitli ticari firmalardan satın alınmıştır. Tüm çözücüler Merck firmasından satın alınmıştır ve kullanımdan önce destillenmiştir. Reaksiyonlar azot gazı atmosferinde nemsiz ortamda gerçekleştirilmiştir. Ftalosiyaninlerin sentezinde Schlenk sistemi kullanılmıştır. Proton ve karbon nükleer manyetik rezonans spektrumları $\left({ }^{1} \mathrm{H}\right.$ NMR, ${ }^{13} \mathrm{C}$ NMR), dötero-dimetilsülfoksit içerisinde Bruker Avance III $400 \mathrm{MHz}$ NMR spektrometresi ile alınmıştır. Kimyasal kayma $(\delta)$ değerleri ppm, bağlanma sabiti değerleri $(J)$ ise $\mathrm{Hz}$ (Hertz) cinsinden verilmiştir. Infrared spektrumları (IR) ATR tekniği kullanılarak Perkin Elmer 1600 Fourier TransformInfrared (FT-IR-ATR) cihazı ile alınmıştır. Elektronik absorpsiyon spektrumları (UV-Vis) dimetilsülfoksit (DMSO) çözücüsü içerisinde, oda sicaklığında, 1 cm'lik yol uzunluğuna sahip küvetler kullanılarak Perkin Elmer Lambda 25 spektrofotometresi ile alınmıştır. Kütle 
spektrumları (MS) için Bruker Microflex LT MALDI-TOF MS spektrometre cihazı kullanılmıştır. Elementel analiz Costech ESC 4010 cihazı kullanılarak gerçekleştirilmiştir. Erime noktaları Barnstead electro thermal 9200 cihazı ile tayin edilmiştir.

$\mathrm{Bu}$ çalışmada kloroform $\left(\mathrm{CHCl}_{3}\right)$, diklorometan $\left(\mathrm{CH}_{2} \mathrm{Cl}_{2}\right)$, tetrahidrofuran (THF), dimetilsülfoksit (DMSO), dimetilformamid (DMF), metanol $(\mathrm{MeOH})$, etanol (EtOH), sodyum hidroksit $(\mathrm{NaOH})$, potasyum karbonat $\left(\mathrm{K}_{2} \mathrm{CO}_{3}\right)$, hidroklorik asit ( $\mathrm{HCl}), \quad$ 1,8-diazabisiklo[5.4.0]undek-7-en (DBU), kobalt(II) klorür $\left(\mathrm{CoCl}_{2}\right)$, bakır(II) klorür $\left(\mathrm{CuCl}_{2}\right)$, nikel(II) klorür $\left(\mathrm{NiCl}_{2}\right)$, çinko(II) asetat $\left(\mathrm{Zn}\left(\mathrm{CH}_{3} \mathrm{COO}\right)_{2}\right)$, azot gazı $\left(\mathrm{N}_{2}(\mathrm{~g})\right)$ kimyasalları kullanılmıştır. Reaksiyonların kontrolü ince tabaka kromatografisi (TLC) ile yapılmıştır.

\subsection{Orijinal bileşiklerin sentezi}

\subsection{1. (E)-3-(3-hidroksifenil)-1-(2,4,6-}

trimetoksifenil)prop-2-en-1-one (1) bileşiğinin sentezi

2,4,6-trimetoksiasetofenon ( $0.5 \mathrm{~g}, 0.0024 \mathrm{~mol})$ ve 3-hidroksibenzaldehit $(0.29 \mathrm{~g}, 0.0024 \mathrm{~mol}) 10 \mathrm{~mL}$

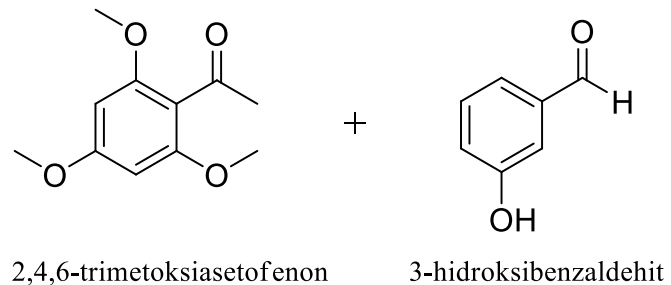

Şekil 1. 1 Numaralı bileşiğe ait sentez şeması

\subsection{2. (E)-4-(3-(3- $(2,4,6-$ \\ trimetoksifenil)akriloil)fenoksi)ftalonitril (3) bileşiğinin sentezi}

25 mL'lik bir reaksiyon balonuna 1 nolu hidroksil bileşiği (3.00 g, $0.0095 \mathrm{~mol})$, 4-nitro ftalonitril (2) $(1.65 \mathrm{~g}, 0.0095 \mathrm{~mol})$ ve $8 \mathrm{~mL}$ dimetilformamid (DMF) eklendi ve $60{ }^{\circ} \mathrm{C}^{\prime} \mathrm{de}$ yarım saat karıştırıldı. Süre sonunda çözeltiye susuz potasyum karbonat $\left(\mathrm{K}_{2} \mathrm{CO}_{3}\right)(3.93 \mathrm{~g}, 0.029 \mathrm{~mol}) 15$ dakika aralıklarla eşit kısımlar halinde ilave edildi. Oluşan karışım azot gazı ( $\left.\mathrm{N}_{2}(\mathrm{~g})\right)$ atmosferi altında $60{ }^{\circ} \mathrm{C}^{\prime}$ de 5 gün karıştırıldı. Reaksiyon tamamlanınca karışım subuz karışımı içerisine döküldü ve karıştırıldı, çöken ham ürün süzülerek ayrıldı ve etanolden kristallendirilerek saflaştırıldı (3). Gerçekleştirilen reaksiyon Şekil 2'de gösterilmiştir. Verim: $3.37 \mathrm{~g}$ $\left(\%\right.$ 80). Erime noktas $1=90-92{ }^{\circ} \mathrm{C}$. FT-IR $\left(\mathrm{KBr}, \mathrm{cm}^{-}\right.$ ${ }^{1}$ ): 3078 (Ar-H), 2942-2841 (Alif. C-H), 2233 etanol (EtOH) içerisinde çözüldü. Bu karışıma oda sıcaklığında $5 \mathrm{~mL} \% 40$ 'lık sodyum hidroksit $(\mathrm{NaOH})$ çözeltisi ilave edildi. Oluşan karışım oda sıcaklığında 1 gün karıştırıldı. Süre sonunda TLC kontrolü yapılarak reaksiyon sonlandırıldı. Reaksiyon sonucunda elde edilen karışım, su-buz karışımı içerisine dökülerek sarı renkli katı ham ürün çökene kadar hidroklorik asit $(\mathrm{HCl})$ ile asitlendirildi. Elde edilen katı ham ürün süzülerek ayrıldı, kurutuldu. Ham ürün 1:1 etanol/su karışımı kullanılarak kristallendirildi ve ürün saf halde elde edildi. Gerçekleştirilen reaksiyon Şekil 1'de gösterilmiştir. Verim: $0.61 \mathrm{~g} \mathrm{( \%} \mathrm{83).} \mathrm{Erime} \mathrm{noktas1}$ $=66-69^{\circ} \mathrm{C}$. FT-IR $\left(\mathrm{KBr}, \mathrm{cm}^{-1}\right): 3219(-\mathrm{OH}), 3051$ (Ar-H), 2937-2837 (Alif. C-H), 1680, 1616, 1577, 1410, 1227, 1126, 974, 779 ; ${ }^{1} \mathrm{H}$ NMR (400 MHz, $\left.\mathrm{CDCl}_{3}-\mathrm{DMSO}-\mathrm{d}_{6}\right) \delta=9.64(-\mathrm{OH}), 7.19(\mathrm{~m}, 1 \mathrm{H})$, 7.13 (d, J=16 Hz., 1H), 7.04 (br, 1H), 6.96 (s, 1H), $6.83(\mathrm{~m}, 2 \mathrm{H}), 6.29(\mathrm{br}, 2 \mathrm{H}), 3.84\left(\mathrm{~s},-\mathrm{OCH}_{3}, 3 \mathrm{H}\right)$, $3.72\left(\mathrm{~s},-\mathrm{OCH}_{3}, 6 \mathrm{H}\right) ;{ }^{13} \mathrm{C} \mathrm{NMR}\left(100 \mathrm{MHz}, \mathrm{CDCl}_{3}-\right.$ DMSO-d 6 ) $\delta=$ (193.6), (162.4), (158.5), (158.2), (144.2), (136.0), (130.3), (129.2), (119.9), (118.1), (115.0), (111.4), (91.5), (56.2), (55.9) ; MS (ESI), $(\mathrm{m} / \mathrm{z})$ : Teorik: 340.16 ; Deneysel: $341.18[\mathrm{M}+\mathrm{H}]^{+}$; Kapal1 Formül: $\mathrm{C}_{18} \mathrm{H}_{18} \mathrm{O}_{5}$; (Elementel Analiz) Teorik: (C: 68.78) (H: 5.77) (O: 25.45) ; Deneysel: (C: 68.76) (H: 5.81) (O: 25.42).

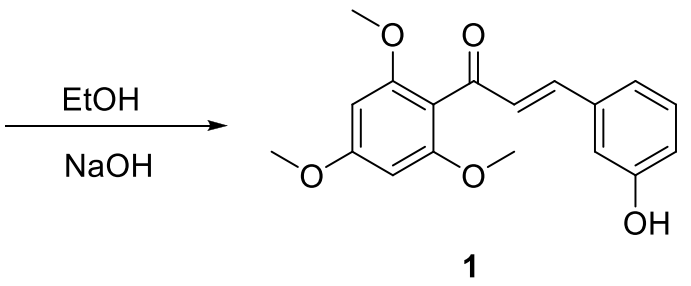

$(\mathrm{C} \equiv \mathrm{N}), 1649,1585,1413,1227,1123,976,810$, 791; ${ }^{1} \mathrm{H}$ NMR $\left(400 \mathrm{MHz}, \mathrm{DMSO}-\mathrm{d}_{6}\right) \delta=7.76(\mathrm{~d}$, $\mathrm{J}=8 \mathrm{~Hz} .1 \mathrm{H}), 7.49(\mathrm{~m}, 2 \mathrm{H}), 7.38(\mathrm{~d}, \mathrm{~J}=16 \mathrm{~Hz} .1 \mathrm{H}$, $A B$ sisteminin $B$ parças 1$), 7.30(\mathrm{~m}, 3 \mathrm{H}), 7.09$ (m, $1 \mathrm{H}), 6.98(\mathrm{~d}, \mathrm{~J}=16 \mathrm{~Hz} .1 \mathrm{H}, \mathrm{AB}$ sisteminin A parçası), $6.17(\mathrm{~s}, 2 \mathrm{H}), 3.87(\mathrm{~s}, 3 \mathrm{H}), 3.79(\mathrm{~s}, 6 \mathrm{H})$, $2.12(\mathrm{~s}, 6 \mathrm{H}) ;{ }^{13} \mathrm{C}$ NMR $(100 \mathrm{MHz}$, DMSO-d 6$) \delta=$ (193.4), (162.7), (161.4), (159.0), (154.0), (141.5), (138.1), (135.5), (131.0), (130.5), (126.1), (121.9), (121.7), (121.6), (119.9), (117.7 (CN)), (115.2 (CN)), (114.9), (111.5), (109.2), (90.7), (56.0), (55.5) ; MALDI-TOF-MS (m/z): Teorik: 440.43; Deneysel: $441.14[\mathrm{M}+\mathrm{H}]^{+}$; Kapalı Formül: $\mathrm{C}_{26} \mathrm{H}_{20} \mathrm{~N}_{2} \mathrm{O}_{5}$; (Elementel Analiz) Teorik: (C: 70.90) (H: 4.58) (N: 6.36) (O: 18.16) ; Deneysel: (C: 70.93) (H: 4.57) (N: 6.39) (O: 18.12). 
<smiles>COc1cc(OC)c(C(=O)/C=C/c2cccc(O)c2)c(OC)c1</smiles>

Şekil 2. 3 Numaralı bileşiğe ait sentez şeması (i: $\mathrm{N}_{2}(\mathrm{~g}), 60{ }^{\circ} \mathrm{C}$, kuru DMF, kuru $\mathrm{K}_{2} \mathrm{CO}_{3}$ )

\subsubsection{Metalsizftalosiyanin (4) sentezi}

(3) Nolu bileşik (250 mg, $0.57 \mathrm{mmol})$, DBU (4 damla) ve kuru $n$-pentanol $(6 \mathrm{~mL})$ karışımı Schlenk tüpü içerisine eklendi $160 \quad{ }^{\circ} \mathrm{C}^{\prime} \mathrm{de} \quad \mathrm{N}_{2}(\mathrm{~g})$ atmosferinde 1 gün boyunca karıştırıldı. Reaksiyon sonunda karışım üzerine etanol ilave edildi ve geri soğutucu altında 4 saat reflux edildi. Çöken ham ürün süzülerek ayrıldı ve düşük basınç altında desikatörde kurutuldu. Daha sonra ham ürün kolon kromatografisi yöntemi ile saflaştırıldı [kloroform:metanol (100:4)]. Gerçekleştirilen reaksiyon Şekil 3'de gösterilmiştir. Verim: $79 \mathrm{mg}$ $\left(\%\right.$ 31). Erime noktas $1=>250{ }^{\circ} \mathrm{C}$. FT-IR $\left(\mathrm{KBr}, \mathrm{cm}^{-}\right.$ $\left.{ }^{1}\right)$ : $3287(\mathrm{~N}-\mathrm{H}), 3067,2994,2931,2837,1575$, 1457, 1228, 1119, 1013, 977, 815, 751; ${ }^{1} \mathrm{H}$ NMR (400 MHz, DMSO-d 6 ) $\delta=7.80-7.48(\mathrm{~m}, 8 \mathrm{H}, \mathrm{ArH})$, 7.40-6.81 (m, 28H, ArH), 6.31-5.97 (m, 8H, ArH), 3.71 (s, 12H, -OMe), 3.62 (s, 24H, -OMe) ; UVVis (DMSO): $\lambda_{\max }, \mathrm{nm}: 703,666,388$; MALDITOF-MS $(\mathrm{m} / \mathrm{z})$ : Teorik: 1763.81 ; Deneysel: $1917.99[\mathrm{M}+\mathrm{DHB}]^{+}$.

\subsubsection{Periferal tetra sübstitüe metalli \\ ftalosiyaninlerin $(5,6,7$ ve 8) genel sentezi}

(3) Nolu bileşik ( $250 \mathrm{mg}, 0.57 \mathrm{mmol}$ ), ilgili susuz metal tuzlar1 $\left[\left(\mathrm{M}=\mathrm{CoCl}_{2}(36.40 \mathrm{mg}, 0.28 \mathrm{mmol})\right.\right.$; $\mathrm{CuCl}_{2}(37.67 \mathrm{mg}, 0.28 \mathrm{mmol}) ; \mathrm{M}=\mathrm{NiCl}_{2}(36.30$ $\mathrm{mg}, 0.28 \mathrm{mmol}) ; \mathrm{M}=\mathrm{Zn}\left(\mathrm{CH}_{3} \mathrm{COO}\right)_{2}(51.35 \mathrm{mg}$, $0.28 \mathrm{mmol})]$, kuru $n$-pentanol $(6 \mathrm{~mL})$ ve DBU (4 damla), Schlenk tüpü içerisinde $160^{\circ} \mathrm{C}$ 'de $\mathrm{N}_{2}(\mathrm{~g})$ atmosferinde 1 gün boyunca karıştırıldılar. Süre tamamlandıktan sonra karışımlara etanol ilave edildi ve geri soğutucu altında 4 saat reflux edildi. Çöken ham ürünler süzülerek ayrıldılar ve desikatörde kurutuldular. Daha sonra ham ürünler kolon kromatografisi yöntemi ile saflaştırıldı [kloroform:metanol (100:1.5)]. Gerçekleştirilen reaksiyonlar Şekil 3'de gösterilmiştir.

\subsubsection{Kobalt(II) ftalosiyanin (5)}

Verim: $87 \mathrm{mg}(\% 33)$. Erime noktas $1=>250{ }^{\circ} \mathrm{C}$. FT-IR $\left(\mathrm{KBr}, \mathrm{cm}^{-1}\right)$ : 3064, 2996, 2936, 2841, 1577, 1457, 1224, 1123, 1018, 975, 810, 754; UV-Vis
(DMSO): $\lambda_{\max }, \mathrm{nm}: 673,621,382$; MALDI-TOFMS (m/z): Teorik: 1819.74; Deneysel: 1969.23 $[\mathrm{M}+\mathrm{DHB}-4 \mathrm{H}]^{+}$.

\subsubsection{Bakır(II) ftalosiyanin (6)}

Verim: $92 \mathrm{mg}(\% 35)$. Erime noktas $1=>250{ }^{\circ} \mathrm{C}$. FT-IR $\left(\mathrm{KBr}, \mathrm{cm}^{-1}\right): 3061,2996,2936,2837,1579$, $1455,1224,1016,972,810,746$; UV-Vis (DMSO): $\lambda_{\max }, \mathrm{nm}: 679,619,380$; MALDI-TOFMS (m/z): Teorik: 1825.31; Deneysel: 1979.82 $[\mathrm{M}+\mathrm{DHB}]^{+}$.

\subsubsection{Nikel(II) ftalosiyanin (7)}

Verim: $89 \mathrm{mg}(\% 34)$. Erime noktas $1=>250{ }^{\circ} \mathrm{C}$. FT-IR (KBr, cm $\left.{ }^{-1}\right): 3065,3000,2937,2838,1578$, 1457, 1225, 1018, 974, 810, 751; ${ }^{1} \mathrm{H}$ NMR (400 MHz, DMSO- $\left.\mathrm{d}_{6}\right) \delta=7.85-7.46(\mathrm{~m}, 8 \mathrm{H}, \operatorname{ArH})$, 7.38-7.03 (m, 28H, ArH), 6.21-6.05 (m, 8H, ArH), $3.76(\mathrm{~s}, 12 \mathrm{H},-\mathrm{OMe}), 3.53$ (s, 24H, -OMe) ; UVVis (DMSO): $\lambda_{\max }, \mathrm{nm}: 671,622,379$; MALDITOF-MS $(\mathrm{m} / \mathrm{z})$ : Teorik: 1818.41; Deneysel: $1972.01[\mathrm{M}+\mathrm{DHB}]^{+}$.

\subsubsection{4. Çinko(II) ftalosiyanin (8)}

Verim: $98 \mathrm{mg}(\% 38)$. Erime noktas $1=>250{ }^{\circ} \mathrm{C}$. FT-IR $\left(\mathrm{KBr}, \mathrm{cm}^{-1}\right)$ : 3007, 2938, 2839, 1579, 1455, 1226, 1019, 975, 809, 761; ${ }^{1} \mathrm{H}$ NMR $(400 \mathrm{MHz}$, DMSO-d $\left._{6}\right) \delta=7.72-7.40$ (m, 8H, ArH), 7.35-6.84 (m, 28H, ArH), 6.39-6.15 (m, 8H, ArH), 3.70 (s, $12 \mathrm{H},-\mathrm{OMe}), 3.35$ (s, 24H, -OMe) ; UV-Vis (DMSO): $\lambda_{\max }, \mathrm{nm}: 672,618,380$; MALDI-TOFMS (m/z): Teorik: 1828.21; Deneysel: 1982.21 $[\mathrm{M}+\mathrm{H}+\mathrm{DHB}]^{+}$. 


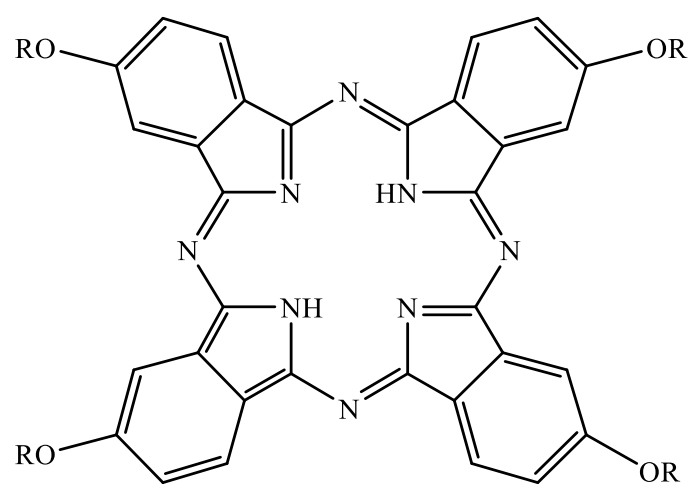

4

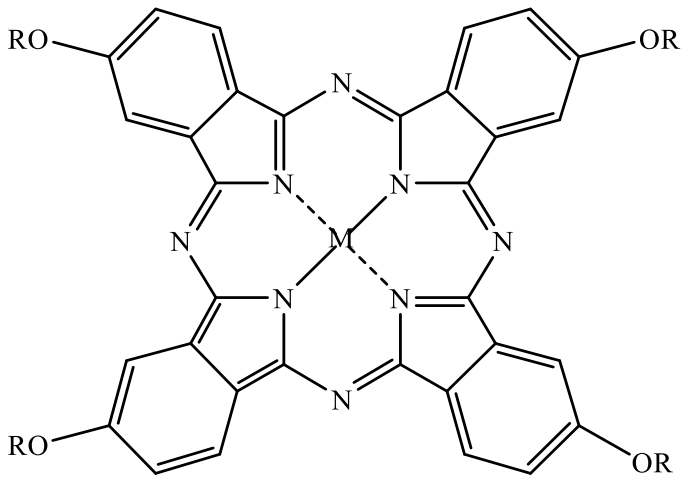

Complex: $\mathbf{5}, \mathbf{6}, \quad \mathbf{7}, \mathbf{8}$

$\mathrm{M}: \quad \mathrm{Co}, \mathrm{Cu}, \mathrm{Ni}, \mathrm{Zn}$
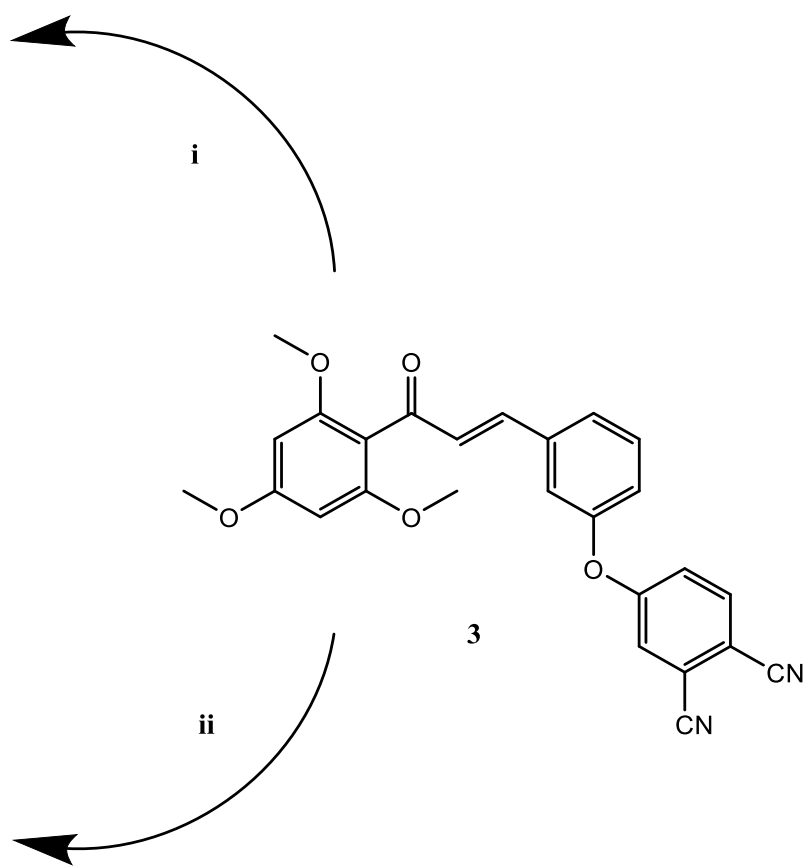<smiles>[R][X]=C(C=Cc1cccc(C)c1)c1c(OC)cc(OC)cc1OC</smiles>

$\mathbf{i}=$ n-pentanol, $\mathrm{DBU}, 160^{\circ} \mathrm{C}, \mathrm{N}_{2}(\mathrm{~g})$

ii $=$ n-pentanol, $\mathrm{DBU}, 160{ }^{\circ} \mathrm{C}, \mathrm{N}_{2}(\mathrm{~g}), \mathrm{CoCl}_{2}, \mathrm{CuCl}_{2}, \mathrm{NiCl}_{2}, \mathrm{Zn}\left(\mathrm{CH}_{3} \mathrm{COO}\right)_{2}$

Şekil 3. 4, 5, 6, 7 ve 8 Numaralı bileşiklere ait sentez şeması

\section{Bulgular ve tartışma}

\subsection{Sentez}

Sentez çalışması özetle üç basamaktan oluşmaktadır. Bu basamaklar 1, 3, 4, 5, 6, 7 ve 8 orijinal bileşikleri için Şekil 1, Şekil 2 ve Şekil 3'de sentez şeması olarak gösterilmiştir.

(-) Sentez çalışmasının ilk basamağında 2,4,6trimetoksiasetofenon ile 3-hidroksibenzaldehit arasındaki reaksiyon sonucu hidroksil bileşiği (1) $\% 83$ verimle saf olarak elde edilmiştir.

(--) İkinci basamakta hidroksil bileşiği (1) ile 4nitroftalonitril (2) arasındaki reaksiyon sonucu $(E)$ 4-(3-(3- $(2,4,6-$

trimetoksifenil)akriloil)fenoksi)ftalonitril bileşiği sentezlenmiştir. 3 Bileşiği kristalizasyon yöntemi ile saflaştırılmıştır ve $\% 80$ verimle elde edilmiștir.

(---) Sentez çalışmasının son basamağında ise $\mathbf{3}$ bileşiğinin, n-pentanol, DBU ve çeşitli metal tuzlar1 $\left(\mathrm{CoCl}_{2}, \mathrm{CuCl}_{2}, \mathrm{NiCl}_{2}, \mathrm{Zn}\left(\mathrm{CH}_{3} \mathrm{COO}\right)_{2}\right)$ ile siklotetramerizasyon reaksiyonu sonucu periferal tetra-substitue metalsiz $\left(\mathrm{H}_{2} \mathrm{Pc} \quad 4\right)$ ve metalli ftalosiyaninler $(\mathrm{Co}(\mathrm{II}) \mathrm{Pc} 5, \mathrm{Cu}(\mathrm{II}) \mathrm{Pc}$ 6, Ni(II)Pc 7, $\mathrm{Zn}(\mathrm{II}) \mathrm{Pc}$ 8) elde edilmiştir. Sentezlenen komplekslerin saflaştırılması kolon kromatografisi yöntemi ile gerçekleştirilmiştir. Elde edilen ftalosiyanin komplekslerinin hepsi yaygın organik çözücüler içerisinde çözünmüştür ve bu çalışmada tercih edilen dimetilsülfoksit (DMSO) çözücüsü içerisinde agregasyon göstermemiştir. 


\subsection{Karakterizasyon}

Çalışmanın karakterizasyon basamağında öncelikle elde edilen orijinal bileşiklerin $(\mathbf{1}, \mathbf{3}, \mathbf{4}, \mathbf{5}$, 6, 7 ve 8) infrared (IR), proton nükleer manyetik rezonans ( ${ }^{1} \mathrm{H}$ NMR), karbon nükleer manyetik rezonans $\left({ }^{13} \mathrm{C}\right.$ NMR), kütle (MS) ve elektronik absorpsiyon (UV-Vis) spektrumları alınmıştır. Elementel analiz orijinal bileşiklerin yapısal karakterizasyonunu desteklemek amaciyla gerçekleştirilmiştir. 1, 3, 4, 5, 6, 7 ve 8 Bileşikleri için spektral karakterizasyon sonuçları aşağıda sırası ile yorumlanmıştır.

(-) 1 Bileşiğinin infrared spektrumunda $3219 \mathrm{~cm}^{-1}$ de $-\mathrm{OH}$ grubuna ait gerilim titreşimi gözlemlenmiştir. 1 Bileşiği için infrared spektrumu yönünden en karakteristik pik -OH (hidroksil) gerilim titreşimi olduğundan bu gözlem bileşiğin başarılı şekilde sentezlendiğini desteklemektedir. 1 Bileşiğinin proton nükleer manyetik rezonans spektrumunda $\delta=9.64(-\mathrm{OH}), 7.19(\mathrm{~m}, 1 \mathrm{H}), 7.13$ (d, $J=16 \mathrm{~Hz} ., 1 \mathrm{H}), 7.04$ (br, 1H), $6.96(\mathrm{~s}, 1 \mathrm{H}), 6.83$ $(\mathrm{m}, 2 \mathrm{H}), 6.29(\mathrm{br}, 2 \mathrm{H}), 3.84\left(\mathrm{~s},-\mathrm{OCH}_{3}, 3 \mathrm{H}\right), 3.72(\mathrm{~s}$, $\left.-\mathrm{OCH}_{3}, 6 \mathrm{H}\right)$ protonlar1 gözlemlenmiştir, bileşiğin toplam hidrojen atomu sayıs ile spektrumunun uyum içerisinde olması yapısını doğrulamaktadır. Bileşiğin karbon sayısı ile karbon nükleer manyetik rezonans spektrumu da uyum içerisindedir ve bu uyum bileşiğin doğru şekilde elde edildiğini göstermektedir. 1 Nolu bileşik için alınan kütle spektrumunda $\mathrm{m} / z=341.18 \quad[\mathrm{M}+\mathrm{H}]^{+}$'de gözlemlenen moleküler iyon piki sentezlenen yeni bileşiğin teorik olarak hesaplanan mol kütlesi ile uyumludur ayrıca elde edilen ürünün elementel analiz sonuçları kütle spektrumu ile birlikte bileşiğin $\mathrm{C}_{18} \mathrm{H}_{18} \mathrm{O}_{5}$ olan kapalı formülünü doğrulamaktadır.

(--) Sentezi gerçekleştirilen $\mathbf{3}$ nolu ftalonitril bileşiğinin infrared spektrumunda $2233 \mathrm{~cm}^{-1}$ 'de nitril grubuna ait pik gözlemlenmiştir. 3 Bileşiği için infrared spektrumu yönünden en karakteristik pik -CN (nitril) gerilim titreşimi olduğundan bu gözlem bileşiğin başarılı şekilde sentezlendiğini desteklemektedir. 3 Nolu bileşiğin proton nükleer manyetik rezonans spektrumunda $\delta=7.76-6.17$ ppm aralığında aromatik protonlara ait ve 3.87$2.12 \mathrm{ppm}$ aralığında ise alifatik protonlara ait kimyasal kayma değerleri gözlemlenmiştir. Sentezlenen bileşiğin proton nükleer manyetik rezonans spektrumu bileşiğin yapısı ile uyum içerisindedir. 3 Nolu bileşiğin karbon nükleer manyetik rezonans spektrumunda sirası ile 115.2 ve 117.7 ppm'de -CN (nitril) grubunun karbon atomlarına ait piklerinin gözlemlenmesi önerilen yeni dinitril bileşiğinin başarılı şekilde sentezlendiğini göstermektedir. Son olarak 3 nolu bileşik için alınan kütle spektrumunda $\mathrm{m} / \mathrm{z}=$ $441.14[\mathrm{M}+\mathrm{H}]^{+}$'de gözlemlenen moleküler iyon piki sentezlenen yeni bileşiğin teorik olarak hesaplanan mol kütlesi ile uyumludur. Kütle spektrumu sonucu ile 3 bileşiğinin elementel analiz sonuçları uyum içerisindedir ve her ikisi birden bileşiğin başarılı şekilde elde edildiğinin kanıtıdır.

(---) Değerlendirmede bütünlük oluşturmak adına aşağıdaki her bir paragrafta $4,5,6,7$ ve $\mathbf{8}$ komplekslerinin sirası ile infrared (IR), kütle (MS), elektronik absorpsiyon (UV-Vis), proton ve karbon nükleer manyetik rezonans $\left({ }^{1} \mathrm{H}\right.$ NMR ve ${ }^{13} \mathrm{C}$ NMR) spektrumları birlikte yorumlanmıştır.

$\mathrm{H}_{2} \mathrm{Pc}$ (4) Bileşiğinin infrared spektrumunda, (E)-4(3-(3- $(2,4,6-$

trimetoksifenil)akriloil)fenoksi)ftalonitril

bileşiğinin $2233 \mathrm{~cm}^{-1}$ 'de görülen nitril grubuna ait pik kaybolmuştur. Ayrıca $\mathrm{H}_{2} \mathrm{Pc}$ (4) bileşiğinin infrared spektrumunda $3287 \mathrm{~cm}^{-1}$ 'de halka içi N-H grubuna ait pik ortaya çıkmıştır. Gözlemlenen bu farklılıklar (3) bileşiği üzerinden metalsiz ftalosiyanin (4) bileşiğinin sentezlendiğini desteklemektedir. Sentezi gerçekleştirilen kobalt(II) ftalosiyanin (5), bakır(II) ftalosiyanin (6), nikel(II) ftalosiyanin (7) ve çinko(II) ftalosiyanin (8) bileşiklerinin infrared spektrumları birbirine çok benzerdir. Her dört ftalosiyanin bileşiğinin infrared spektrumunda $\mathbf{3}$ bileşiğinin nitril grubuna ait $2233 \mathrm{~cm}^{-1}$ de görülen pikin kaybolması 3 bileşiği üzerinden bahsedilen metalli ftalosiyanin komplekslerinin elde edildiğini göstermektedir.

Ftalosiyaninler oldukça büyük molekül ağırlığına sahip bileşiklerdir ayrıca metanol gibi çözücülerde düşük çözünürlük göstermektedirler. $\mathrm{Bu}$ sebeplerden dolayı kütle spektrumları küçük molekül ağırlıklı ve çözünürlüğ̈̈ yüksek organik bileşiklere göre daha zor yorumlanmaktadır. Fragment analizi ile sonuç alınması büyük molekül ağırlıkları sebebiyle çoğu zaman mümkün olmamaktadır. Sentezi gerçekleştirilen 4, 5, 6, 7 ve 8 bileşiklerinin MALDI-TOF yöntemiyle alınan kütle spektrumlarında matriks olarak dihidroksibenzoikasit (DHB) kullanılmıştır (Göksoy vd; 2016). DHB bileşiğinin mol kütlesi $154.12 \mathrm{~g} / \mathrm{mol}$ dür. Metalsiz ftalosiyanin (4) bileşiği için kütle spektrumunda $m / z: 1917.99[\mathrm{M}+\mathrm{DHB}]^{+}$ iyon piki gözlemlenmiştir ve bu değer (4) bileşiğinin teorik olarak hesaplanan mol kütlesi ile uyum içerisindedir. Sentezi gerçekleştirilen kobalt(II), bakır(II), nikel(II) ve çinko(II) metaloftalosiyaninlerin $(\mathbf{5}, \mathbf{6}, \mathbf{7}$ ve 8) teorik olarak hesaplanan mol kütleleri sirası ile 1819.74, 
1979.82, 1818.41 ve $1982.21 \mathrm{~g} / \mathrm{mol}$ dür. $\mathrm{Bu}$ bileşiklerin kütle spektrumları incelendiğinde kobalt(II) ftalosiyanin için $m / z: 1969.23$ [M+DHB4H ${ }^{+}$, bakır(II) ftalosiyanin için $\mathrm{m} / \mathrm{z}: 1979.82$ [M+DHB] $]^{+}$, nikel(II) ftalosiyanin için $m / z: 1972.01$ $[\mathrm{M}+\mathrm{DHB}]^{+}$ve çinko(II) ftalosiyanin için $\mathrm{m} / \mathrm{z}$ : $1982.21[\mathrm{M}+\mathrm{H}+\mathrm{DHB}]^{+}$moleküler iyon pikleri gözlemlenmiştir. $\mathrm{Bu}$ değerler sentezlenen ftalosiyanin komplekslerinin önerilen yapısını desteklemektedir.

Ftalosiyaninlerin elektronik absorpsiyon spektrumları yaklaşı olarak 630-780 nm aralığında keskin bir Q bandı ve yaklaşık olarak 320-380 $\mathrm{nm}$ aralığında zayıf bir B (Soret) band 1 olmak üzere iki kısma ayrilır. Her iki absorpsiyon bandı $\pi-\pi^{*}$ geçişlerinden oluşur. Q bandı ftalosiyaninlerin metal içerip içermediklerine dair bilgi edinmemize yardımc1 olur. Metalli ftalosiyaninlerde ftalosiyanin halkasinda bulunan metal iyonu halka ile dört adet eşdeğer bağ yapar ve simetri $\mathrm{D}_{4 h}$ olarak belirlenir ve bu simetriden dolay1 Q bandı keskin bir pik olarak gözlemlenir (Mack vd; 2001). Metalsiz ftalosiyaninlerde simetri $\mathrm{D}_{2 h}$ olarak belirlenir ve simetrinin düşmesi, Q bandının yarılarak iki eşit pik olarak gözlemlenmesine neden olur (Kobayashi vd; 2000). 4, 5, 6, 7 ve 8 Bileşiklerinin $25^{\circ} \mathrm{C}$ 'de DMSO içinde $1 \times 10^{-5} \mathrm{M}$ konsantrasyonda kaydedilen elektronik absorpsiyon spektrumları Şekil 4'te görülmektedir.
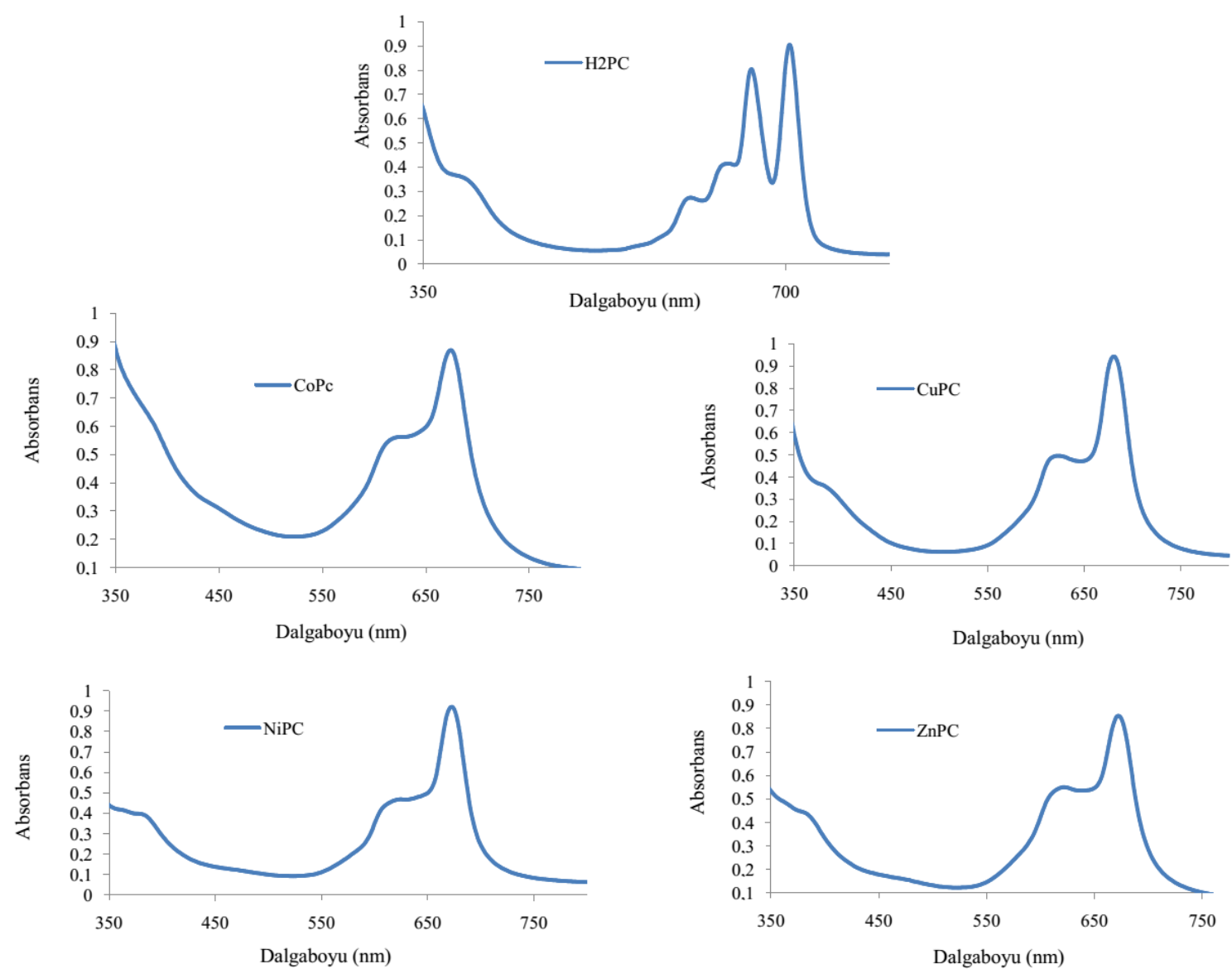

Şekil 4. $\mathrm{H} 2 \mathrm{Pc}$ (4), $\mathrm{Co}(\mathrm{II}) \mathrm{Pc}(5), \mathrm{Cu}(\mathrm{II}) \mathrm{Pc}(6), \mathrm{Ni}(\mathrm{II}) \mathrm{Pc}(7)$ ve $\mathrm{Zn}(\mathrm{II}) \mathrm{Pc}$ (8) Bileşiklerinin oda sıcaklığında DMSO içinde $1 \times 10^{-5} \mathrm{M}$ konsantrasyonda kaydedilen UV-Vis spektrumları

Metalsiz ftalosiyanin (4) bileşiğinin elektronik absorpsiyon spektrumunda $\mathrm{Q}$ band absorpsiyonları $\lambda_{\max }: 703$ ve $666 \mathrm{~nm}$ 'de ve B bandabsorpsiyonu ise $\lambda_{\max }: \quad 388 \mathrm{~nm}$ 'de gözlemlenmiştir. Bileşiğin elektronik absorpsiyon spektrumunda 703 ve 666 nm civarında görülen yarılmış Q bandına sahip olmas1 kavitesine herhangi bir metal iyonu bağlamadığını göstermektedir. Kobalt(II), bakır(II), nikel(II) ve çinko(II) metaloftalosiyaninlerin $(\mathbf{5}, \mathbf{6}, \mathbf{7}$ ve $\mathbf{8})$ elektronik absorpsiyon spektrumlarında sirasiyla 673, 679, 671 ve 672 nm'de gözlenen pikler ikiye yarılmamış keskin Q bandına aittir. B bandları kobalt(II) ftalosiyanin (5) için 382 nm'de, bakır(II) ftalosiyanin (6) için $380 \quad \mathrm{~nm}$ 'de, nikel(II) ftalosiyanin (7) için $379 \mathrm{~nm}$ 'de ve çinko(II) ftalosiyanin (8) için $380 \mathrm{~nm}$ 'de gözlemlenmiştir. 5, 6, 7 ve 8 Komplekslerinin UV-Vis spektrumlarında tek ve keskin bir Q bandına sahip olması, metalli 
ftalosyaninlerin $\mathrm{D}_{4 h}$ simetrisi ve genel UV-Vis spektrumları ile uyumludur.

Paramanyetik kobalt(II) ve bakır(II) iyonlarını bulundurmaları sebebiyle $\mathbf{5}$ ve $\mathbf{6}$ numaralı ftalosiyanin komplekslerinin proton ve karbon nükleer manyetik rezonans spektrumları alınamamaktadır (Değirmencioğlu vd; 2012). (3) Nolu bileşik üzerinden sentezlenen $\mathrm{H}_{2} \mathrm{Pc}$ bileşiğinin (4) proton nükleer manyetik rezonans spektrumunda metalsiz ftalosiyaninin (4) halka içinde bulunan protonlarına ait sinyal, ölçümler esnasında hazırlanan çözeltilerin yüksek konsantrasyonlu olmas1 ve bu konsantrasyonda molekülün muhtemel agregasyonu sebebiyle gözlenememiştir (Değirmencioğlu vd; 2011). Bileşiğin ${ }^{1} \mathrm{H}$ NMR spektrumunda beklendiği gibi başlangıç (3) bileşiğinin ${ }^{1} \mathrm{H}$ NMR spektrumundan çok farklı olmamakla birlikte aromatik protonlara ait kimyasal kayma değerleri 7.80-5.97 ppm aralığında, alifatik protonlara ait kimyasal kayma değerleri ise sirasiyla 3.71 ve 3.62 ppm'de gözlemlenmiştir. Nikel(II) ftalosiyanin (7) bileşiğinin dötoro DMSO'da alınan proton nükleer manyetik rezonans spektrumunda kimyasal kayma değerleri aromatik protonlar için 7.85-6.05 ppm aralığ olarak, alifatik protonlar içinse $3.76 \mathrm{ppm}$ ve $3.53 \mathrm{ppm}$ olarak belirlenmiştir. Bu değerler aynı proton sayısına sahip çinko(II) ftalosiyanin (8) için 7.72-6.15 ppm aralığ $(\mathrm{ArH}), 3.70 \mathrm{ppm}(-\mathrm{OMe})$ ve $3.35 \mathrm{ppm}(-\mathrm{OMe})$ olarak değişiklik göstermiştir. Proton nükleer manyetik rezonans spektrum sonuçları 4, 7 ve 8 bileşiklerinin belirlenen yapıları ile uyum içerisindedir.

\section{Sonuçlar}

$\mathrm{Bu}$ çalışma kapsamında sentez ve karakterizasyonu gerçekleştirilen hidroksil bileşiği 1, ftalonitril bileşiği $\mathbf{3}$, metalsiz ve metalli ftalosiyanin kompleksleri 4, 5, 6, 7 ve 8 orijinal bileşikler olup literatürde bulunmamaktadır. Ftalonitril bileşiği 3 üzerinden metalsiz, diamanyetik nikel(II) ve çinko(II) aynı zamanda paramanyetik kobalt(II) ve bakır(II) merkez metal iyonları içeren periferal tetra substitue ftalosiyanin kompleksleri sentezlenmiştir. Sentezi gerçekleştirilen tüm yeni bileşikler $\left(\mathbf{1}, \mathbf{3}, \mathbf{4}, \mathbf{5}, \mathbf{6}, 7\right.$ ve 8) Infrared, ${ }^{1} \mathrm{H}$ NMR, ${ }^{13} \mathrm{C}$ NMR, MS, elektronik absorpsiyon spektroskopi ve elementel analiz teknikleri kullanılarak karakterize edilmiştir. Sentez ve karakterizasyonu gerçekleştirilen tüm kompleksler $\left(4,5,6,7\right.$ ve 8) kloroform $\left(\mathrm{CHCl}_{3}\right)$, diklorometan $\left(\mathrm{CH}_{2} \mathrm{Cl}_{2}\right)$, tetrahidrofuran (THF), dimetilsülfoksit (DMSO) ve dimetilformamid (DMF) gibi yaygın organik çözücüler içerisinde çözünmektedir ve bu çalışmada tercih edilen dimetilsülfoksit (DMSO) çözücüsü içerisinde agregasyon göstermemektedir. $\mathrm{Bu}$ durum sentez ve karakterizasyonu gerçekleştirilen ftalosiyanin komplekslerini farklı uygulama alanları için çalışılabilir kılmaktadır. Bu çalışma alanları için bir değerlendirme yapıldığında; hem kobalt(II) ftalosiyanin (5) hem de bakır(II) ftalosiyanin (6) bileşikleri için daha ileriki aşamalarda katalizör özellikleri incelenebilir ve ayrı bir çalışma olarak literatüre kazandırılabilir. Substitüe metalli ftalosiyaninlerden çinko ftalosiyaninler PDT için en uygun fotosensitizerlerden biridir. Ayrica literatür incelendiğinde tetra sübstitüe metalsiz ftalosiyaninlerin de fotodinamik terapi için fotosensitizer özelliklerinin incelendiği görülmektedir. Dolayısı ile metalsizftalosiyanin (4) ve çinko(II) ftalosiyanin (8) bileşikleri için daha ileriki aşamalarda fotosensitizer özelliklerinin incelenmesi üzerine farklı bir çalışma gerçekleştirilebilir ve sonuçların olumlu olması durumunda bu çalışma da literatüre kazandırılabilir.

\section{Kaynaklar}

Ao, R., Kümmerl, L. and Haarer, D. (1995). Present limits of data storage using dye molecules in solid matrices. Advanced Materials, 7, 495-499, https://doi.org/10.1002/adma.19950070522

Bekaroğlu, Ö. (1996). Phthalocyanines containing macrocycles applied. Organometallic Chemistry, 10 , https://doi.org/10.1002/(SICI)1099605-622, 0739(199610)10:8<605::AIDAOC527>3.0.CO;2-U

Değirmencioğlu, İ., Bayrak, R., Akcay, H. T., Pişkin, M. and Durmuş, M. (2012). Azine-bridged binuclear metallophthalocyanines functioning photophysical and photochemical-responsive. Dyes and Pigments, 95, 330-337, https://doi.org/10.1016/j.dyepig.2012.05.010

Değirmencioğlu, İ., Bayrak, R., Er, M. and Serbest, K. (2011). New olefinic centred binuclear clamshell type phthalocyanines: design, synthesis, structural characterization, the stability and the change in the electron cloud at olefine-based symmetrical diphthalonitrile fragment by the combined application of UV-Vis electronic structure and theoretical methods. Polyhedron, 30 , https://doi.org/10.1016/j.poly.2011.03.039 1628-1636,

Dini, D., Barthel, M., Schneider, T., Ottmar, M., Verma, S. and Hanack, M. (2003). Phthalocyanines and related compounds as switchable materials upon strong irradiation: the molecular engineering behind the optical limiting effect. Solid State 

$\begin{array}{lcc}\text { Ionics, } & 165, & 289-303, \\ \text { https://doi.org/10.1016/j.ssi.2003.08.046 }\end{array}$

Göksoy, B., Orman, E. B., Kuruca, H., Bulut, M., Durmuş, M. and Özkaya A. R. (2016). Mono and double decker lutetium phthalocyanines bearing iodine groups electrochemical and electrochromic properties. Journal of the Electrochemical Society, 163, 927-936, https://doi.org/10.1149/2.0421610jes

Kluson, P., Drobek, M., Kalaji, A., Zarubova, S., Krysa, J. and Rakusan, J. (2008). Singlet oxygen photogeneration efficiencies of a series of phthalocyanines in well-define $\mathrm{d}$ spectral regions. Journal of Photochemistry and Photobiology A: Chemistry, 199, 267-273, https://doi.org/10.1016/j.jphotochem.2008.06.00 3

Kobayashi, N., Muranaka, A. and Ishii, K. (2000). Symmetry-lowering of the phthalocyanine chromophore by a $\mathrm{C}_{2}$ type axial ligand. Inorganic Chemistry, 39, 2256-2257, https://doi.org/10.1021/ic9914950

Mack, J. and Stillman, M. J. (2001). Assignment of the optical spectra of metal phthalocyanines through spectral band deconvolution analysis and zindo calculations. Coordination Chemistry Reviews, 219-221, 993-1032, https://doi.org/10.1016/S0010-8545(01)00394-0
Mutlu, F., Pișkin, M., Canpolat, E. and Öztürk, Ö. F. (2020). The new zinc(II) phthalocyanine directly conjugated with 4-butylmorpholine units: synthesis, characterization, thermal, spectroscopic and photophysical properties. Journal of Molecular Structure, 1201, 127169. https://doi.org/10.1016/j.molstruc.2019.127169

Sarkı, G., Kantekin, H., Yalazan, H., Kahriman, N., Bıyıklığlu, Z. and Serdaroğlu, V. (2019). Synthesis, characterization and electrochemical studies of metal-free and metallophthalocyanines containing two different chalcone units substituted on peripherally positions. Journal of Molecular Structure, 1196, 592-603. https://doi.org/10.1016/j.molstruc.2019.05.123

Ünlü, S., Yaraşır, M. N., Kandaz, M., Koca, A. and Salih, B. (2008). Synthesis, spectroscopy and electrochemical properties of highly soluble fluoro containing phthalocyanines. Polyhedron, 27 , 2805-2810 https://doi.org/10.1016/j.poly.2008.05.036

Yongde, Y. and Zhenguo, J. (2006). Improved photoreceptor decay characteristics of vanadylphthalocyanine films annealed under magnetic field. Journal of Photochemistry and Photobiology A: Chemistry, 179, 348-350, https://doi.org/10.1016/j.jphotochem.2005.09.00 5 